\title{
Animaux transgéniques et oncogenèse
}

De nombreux modèles murins de cancers ont été créés grâce à la production d'animaux transgéniques exprimant un oncogène contrôlé par les régions régulatrices d'un gène spécifique de la différenciation tissulaire. De tels oncogènes ont ainsi une expression ciblée vers le tissu dans lequel sont normalement actives les régions de contrôle utilisées. Certaines combinaisons oncogène/tissu aboutissent au développement de tumeurs qui sont presque toujours clonales, alors que le transgène est exprimé dans toutes les cellules du tissu. Cela souligne l'intervention dans la transformation tumorale d'événements multiples, l'expression de l'oncogène n'étant que l'un d'entre eux. Ces modèles animaux de cancer revêtent une grande importance pour les recherches physiopathologiques et thérapeutiques.

\section{Pierre Gerlinger}

\section{RÉFÉRENCES}

1. Bishop M. Les oncogènes. Pour la Science $1982 ; 55: 28-39$

2. Weinberg $R$. Une base moléculaire du cancer. Pour la Science $1984 ; 75: 12-26$

3. Stéhelin D. Les oncogènes cellulaires, clés de la cancérogenèse. médecine/sciences 1985 ; $1: 12-6$.

4. Croce C, Klein G. Translocations chromosomiques et cancers humains. Pour la Science $1985 ; 91: 42-8$.

5. Kaplan JC, Szajnert MF. Chromosomes et cancer. Le paradigme du lymphome de Burkitt. médecine/sciences $1985 ; 1$ : 17-23.

6. Hunter T. Les protéines des oncogènes. Pour la Science 1984; 84 : 90-101.

7. Barbacid M. Mutagens, oncogenes and cancer. Trends Genet 1986; 2 : 188-92.

\section{ADRESSE}

P. Gerlinger : professeur à la faculté de médecine de Strasbourg. Inserm U. 184, 11, rue

es modalités de la transformation cellulaire ainsi que celles de l'apparition de tumeurs bénignes ou malignes restent encore méconnues. Cependant, la découverte récente des séquences d'oncogènes représente un brin accessible de l'écheveau qui reste à démêler [1-3]. Les proto-oncogènes que nous possédons tous dans notre patrimoine héréditaire ont été identifiés à partir de tissus tumoraux en raison même de leur capacité à transformer des cellules in vitro. Ce sont, pour la plupart, des gènes de facteurs de croissance, des récepteurs cellulaires de ces derniers, des protéines chargées de véhiculer vers le noyau les signaux que reçoit la cellule ou des facteurs contrôlant directement la transcription. Leur expression est importante pour tous les phénomènes de morphogenèse et de différenciation cellulaire au cours de la vie embryonnaire et fœtale. En revanche, leur expression inadéquate a des conséquences dramatiques sur la cinétique et la différenciation cellulaires. Les mécanismes entraînant des désordres de l'expression des proto-oncogènes sont généralement, soit une surexpression par translocation $[4,5]$ dans une zone fortement exprimée, soit une activité différente de la protéine correspondante due à une mutation [6, 7]. En revanche, il apparaît nettement que ces désordres ne sont en général pas la cause directe du phénomène de cancérisation, mais plutôt un facteur favorisant. L'analyse des événements successifs nécessaires à la formation d'une tumeur sera facilitée par l'obtention de modèles expérimentaux où l'on pourra à volonté induire et contrôler l'expression des séquences d'oncogènes dans le type cellulaire de son choix. Ainsi la possibilité de cibler l'expression d'un oncogène, chez un animal transgénique* (note p. 167), nous offre les moyens d'étudier les répercussions de cette expression sur le phénotype cellulaire. 
Tableau 1

PRINCIPALES TUMEURS OBTENUES CHEZ DES SOURIS TRANSGÉNIQUES

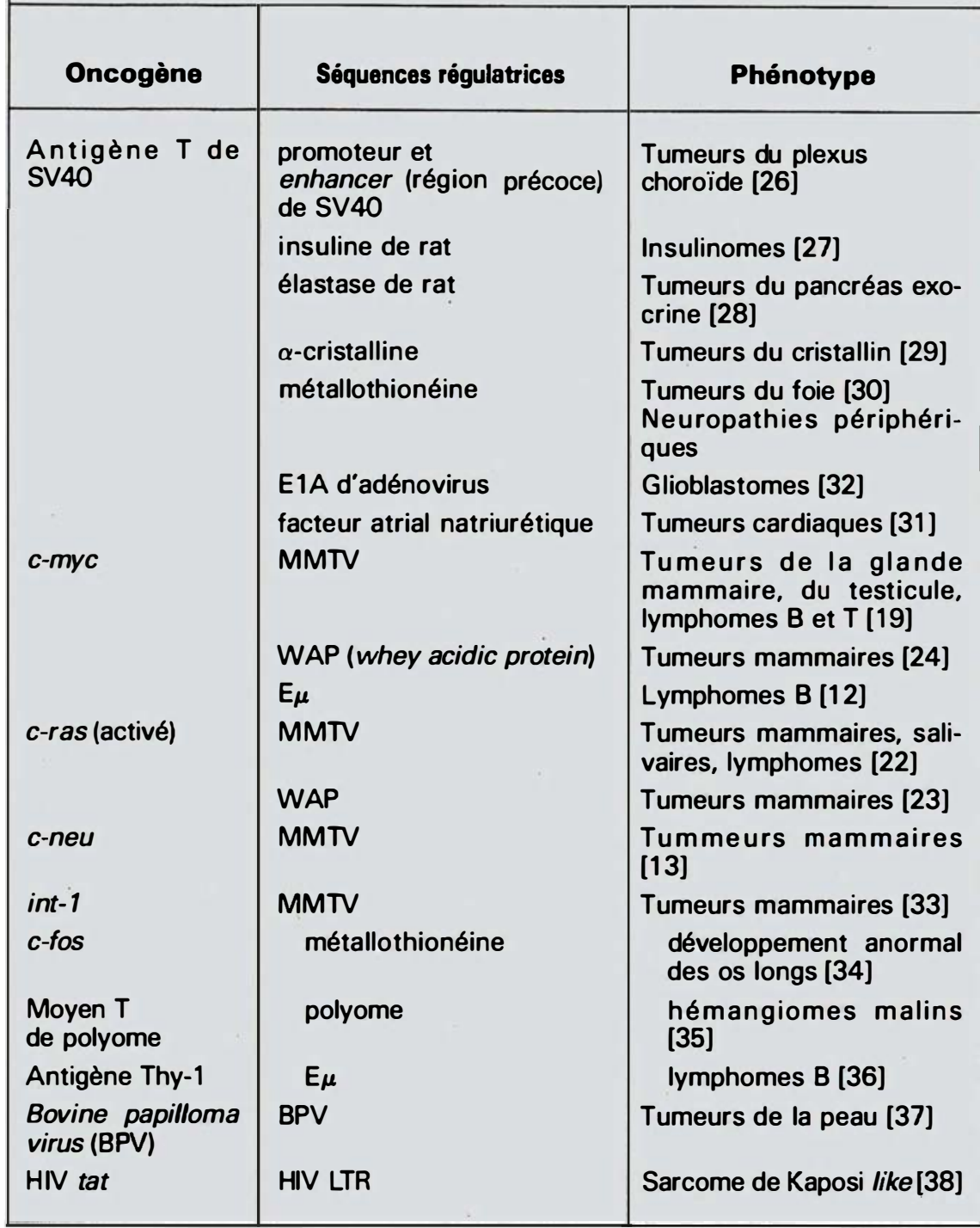

$E \mu=$ enhancer du gène des chaînes lourdes d'immunoglobuline ; MMTV = mouse mammary tumor virus; $B P V=$ bovine papilloma virus ; HN-LTR = long terminal repeat du virus HN.

Dans le cadre de cet article, nous apporterons des éléments de réponse aux questions concernant l'éventail, en terme de type cellulaire, du pouvoir transformant des oncogènes, de la possibilité de leur potentialisation mutuelle, de leur influence sur la croissance, la différenciation et l'activité cellulaires.

Nous tenterons d'exposer et de discu-

* Animal dans le génome duquel a été introduit de manière expérimentale un ou plusieurs gènes $[8,9]$.

$\mathrm{m} / \mathrm{s} n^{\circ} 3$ vol. 5 , mars 89 l'homme, plasmocytome chez la souris) et $T$, car il est placé, par translocation chromosomique, sous le contrôle de séquences régulatrices spécifiques des lymphocytes [5]. La protéine correspondante à l'oncogène $m y c$, sans doute un médiateur nucléaire de facteurs de croissance $[10,11]$, est généralement exprimée au cours du cycle cellulaire, après la transition $\mathrm{G0} / \mathrm{Gl}$. Le niveau d'expression est élevé lors de la prolifération pour décroître au fur et à mesure de la différenciation cellulaire. Un recombinant juxtaposant le enhancer du gène des chaînes lourdes des immunoglobulines et l'oncogène $c-m y c \quad(\mathrm{E} \mu$-myc), intégré dans le génome de souris transgéniques, reproduit expérimentalement la situation rencontrée dans certains lymphomes de Burkitt [11]. De fait, le transgène est exprimé spécifiquement dans les lymphocytes de type B et après un temps de latence relativement court (ll semaines en moyenne), la plupart des animaux transgéniques [13, 15] ont développé une leucémie foudroyante avec hypertrophie des ganglions lymphatiques, de la rate et du thymus. Les cellules transformées, des lymphocytes pré-B et $B$, sont très agressives et envahissent la plupart des tissus après injection à des animaux syngéniques. Bien que tous les lymphocytes B expriment $m y c$, l'analyse des réarrangements des gènes des immunoglobulines démontre une origine mono- ou oligoclonale des tumeurs. Ce résultat est valable, à une exception près ([13], voir plus loin), pour toutes les tumeurs obtenues à ce jour par expression d'oncogènes chez des souris transgéniques. Il confirme la nécessité d'une cascade de plusieurs événements, liés ou non, pour aboutir à l'apparition de cellules tumorales. Le problème reste donc entier : l'expression de myc n'étant pas suffisante pour engendrer la transformation cellulaire, pourquoi cette expression favorise-t-elle une telle fréquence d'apparition de tumeurs? Le stade prénéoplasique chez les jeunes souris $(\mathrm{E} \mu$-myc) offre l'opportunité d'examiner l'influence de $m y c$ sur la différenciation des lymphocytes [14] qui se fait en plusieurs étapes, débutant dans le foie fœtal pour se poursuivre dans la moelle hématopoïétique chez l'adulte. Les 
8. Babinet C, Morello D. Animaux transgéniques: une voie nouvelle pour l'étude du développement. médecine/sciences 1986; 2 : 253-9.

9. Houdebine LM. Les animaux transgéniques. La Recherche 1987 ; 188 : 684-94.

10. Kelly K, Cochran BH, Stiles CD, Leder P. Cell-specific regulation of the $c$-myc gene by lymphocyte mitogens and platelet derived growth factor. Cell 1983 ; 35 : 603-10.

11. Rapp UR, Cleveland JL, Brightman K, Scott A, Ihle JN. Abrogation of IL-2 and IL 3 dependence by recombinant murine retroviruses expressing $v$-myc oncogenes. Nature 1985 ; $917: 434-8$.

12. Adams JM, Harris AW, Pinkert CA, et al. The $c-m y c$ oncogene driven by immonoglobulin enhancers induces lymphoid malignancy in transgenic mice. Nature $1985 ; 318$ : 533-8.

13. Muller WJ, Sinn E, Pattengale PK, Wallace $\mathbf{R}$, Leder $\mathbf{P}$. Single-step induction of mammary adenocarcinoma in transgenic mice bearing the activated $c$-neu oncogene. Cell 1988; 54 : 105-15.

14. Langdon WY, Harris AW, Cory S, Adams JR. The $c$-myc oncogene perturbs B lymphocyte development in $\mathrm{E} \mu$-myc transgenic mice. Cell $1986 ; 47: 11-8$.

15. Klinken SP, Alexander WS, Adams JR. Hemopoietic lineage switch : v-raf oncogene convert $\mathrm{E} \mu$-myc transgenic $\mathrm{B}$ cells into macrophages. Cell $1988 ; 53: 857-67$.

16. Stewart TA, Pattengale PK, Leder P. Spontaneous mammary adenocarcinomas in transgenic mice that carry and express MMTV-myc fusion genes. Cell $1984 ; 38: 627$. 37.

17. Henrard D, Ross SR. Endogenous mouse mammary tumor virus is expressed in several organs in addition to the lactating mammary gland. J. Virol $1988 ; 62$ : 3046-9.

18. Quaife CJ, Pinkert CA, Ornitz DM, Palmiter RD, Brinster RL. Pancreatic neoplasia induced by ras expression in acinar cells of transgenic mice. Cell $1987 ; 48$ : 1023-34.

19. Leder A, Pattengale PK, Kuo A, Stewart TA, Leder P. Consequences of widespread deregulation of the $c-m y c$ gene in transgenic mice : multiple neoplasms and normal development. Cell 1986 ; 45 : 485-95.

20. Land H, Parada LF, Weinberg RA. Tumorigenic conversion of primary embryo fibroblasts requires two cooperating oncogenes. Nature 1983 ; 304 : 596-602.

21. Mougneau E, Glaichenhaus N, Cuzin F. Analyse génétique des étapes précoces de la progression tumorale. médecine/sciences $1985 ; 1: 86-90$.

22. Sinn E, Muller W, Pattengale P, Tepler I, Wallace $R$, Leder $P$. Coexpression of $M M T V / v-r a s$ and $M M T V / c-m y c$ genes in transgenic mice : synergistic action of oncogenes in vivo. Cell 1987; $49: 465-75$

23. Andres AC, Schönenberger CA, Groner B, Hennighausen L, Lemeur M, Gerlinger P. Ha-ras oncogene expression directed by a milk protein gene promoter: tissue specificity, hormonal regulation and tumor induction in transgenic mice. Proc Natl Acad Sci USA 1987 ; 74 : 1299-303. différents stades de cette maturation peuvent être établis par l'analyse de marqueurs de surface et des réarrangements des gènes d'immonoglobulines. A tous les stades du développement, le foie puis la moelle des animaux transgéniques présentent une très large surpopulation polyclonale de cellules de type blastique à des stades pré-B, une diminution des cellules B mûres comme des cellules des lignées érythro- et myélocytaires. La prééminence des cellules pré-B est retrouvée dans la rate, avec cependant une augmentation générale de l'hématopoï̀se alors que les ganglions et le thymus sont normaux (analyse histologique et par cytophotométrie de flux). Ces expériences montrent sans ambiguïté que la surexpression de $m y c$ favorise très nettement la multiplication des précurseurs des lymphocytes B par rapport à leur différenciation, augmentant fortement la proportion de cellules immatures qui restent dans un pool de cellules en prolifération, de ce fait augmentant statistiquement les chances d'apparition des événements successifs nécessaires à la transformation cellulaire. L'expression normalement plus réduite de myc permet, au contraire, de faire pencher la balance dans le sens de la différenciation et de l'arrêt de la prolifération des éléments de cette lignée cellulaire.

Mais là ne s'arrête pas l'apport dû à ce modèle. L'expression d'un oncogène peut-il forcer une cellule à suivre une voie de différenciation inhabituelle? Des lignées de cellules pré$\mathrm{B}$ et $\mathrm{B}$, établies à partir de lymphones dus à l'expression de $\mathrm{E} \mu$-myc, ont été infectées avec un virus contenant l'oncogène raf (oncogène appartenant à la famille des protéines kinases). Une grande proportion des cellules effectivement infectées se différencient secondairement en macrophages, formant des colonies de grandes cellules irrégulières et adhérentes, capables de phagocyter des billes de latex, synthétisant des enzymes catabolitiques, exprimant les oncogènes $c$ - $m y b$ et $c$ - $f m s$, et présentant certains marqueurs de surface spécifiques, toutes caractéristiques des macrophages [15]. Ces cellules ont conservé les réarrangements des gènes d'immonoglobines qui les caractérisaient, continuent à exprimer $m y c$ et sont capables de former des tumeurs chez des souris nude. Ainsi, des lymphocytes $\mathrm{B}$ au terme de leur différenciation sont encore capables de se convertir en un autre type cellulaire en raison de l'expression supplémentaire d'un oncogène particulier !

\section{Ciblage de l'expression d'oncogènes dans le tissu mammaire}

L'oncogène myc tenu pour responsable de tumeurs lymphocytaires «spontanées» ou expérimentales peut-il entraîner la transformation d'autres types cellulaires? En raison même de l'incidence des tumeurs mammaires, il est intéressant de pouvoir créer des modèles expérimentaux permettant l'étude des événements génétiques qui favorisent, accompagnent ou sont directement responsables de la formation des tumeurs. Le gène chimérique comprenant les séquences de contrôle du MMTV (mouse mammary tumor virus) et $m y c$ a été injecté dans le but de provoquer des tumeurs mammaires [16]. En fait, cette construction s'exprime de façon modulable par les glucocorticoïdes et selon les lignées transgéniques, dans plusieurs tissus (figure 1) (la glande mammaire bien entendu, mais aussi la rate, les glandes salivaires, les poumons, les reins, le cerveau, la prostate, les testicules et probablement dans d'autres organes ou tissus non explorés), aussi pourrait-elle servir à établir ce qui pourrait s'appeler le spectre de transformation de $m y c$ [17]. (Il existe en effet de multiples exemples de la variabilité du pouvoir transformant des oncogènes en fonction du type cellulaire où ils s'expriment. Le ciblage de l'expression de $m y c$ dans les cellules acineuses pancréatiques grâce au promoteur de l'élastase est ainsi sans effet [18], alors que dans les mêmes conditions, l'oncogène ras activé est responsable de la transformation rapide des cellules pancréatiques dès le stade fœetal.) A l'âge moyen de 12 mois, les animaux exprimant le transgène $M M T V-m y c$ développent différents types de tumeur : mammaires après plusieurs lactations, testiculaires, lymphocytaires $B$ et $T$, mais jamais pulmonaires, pancréatiques ou salivaires, 


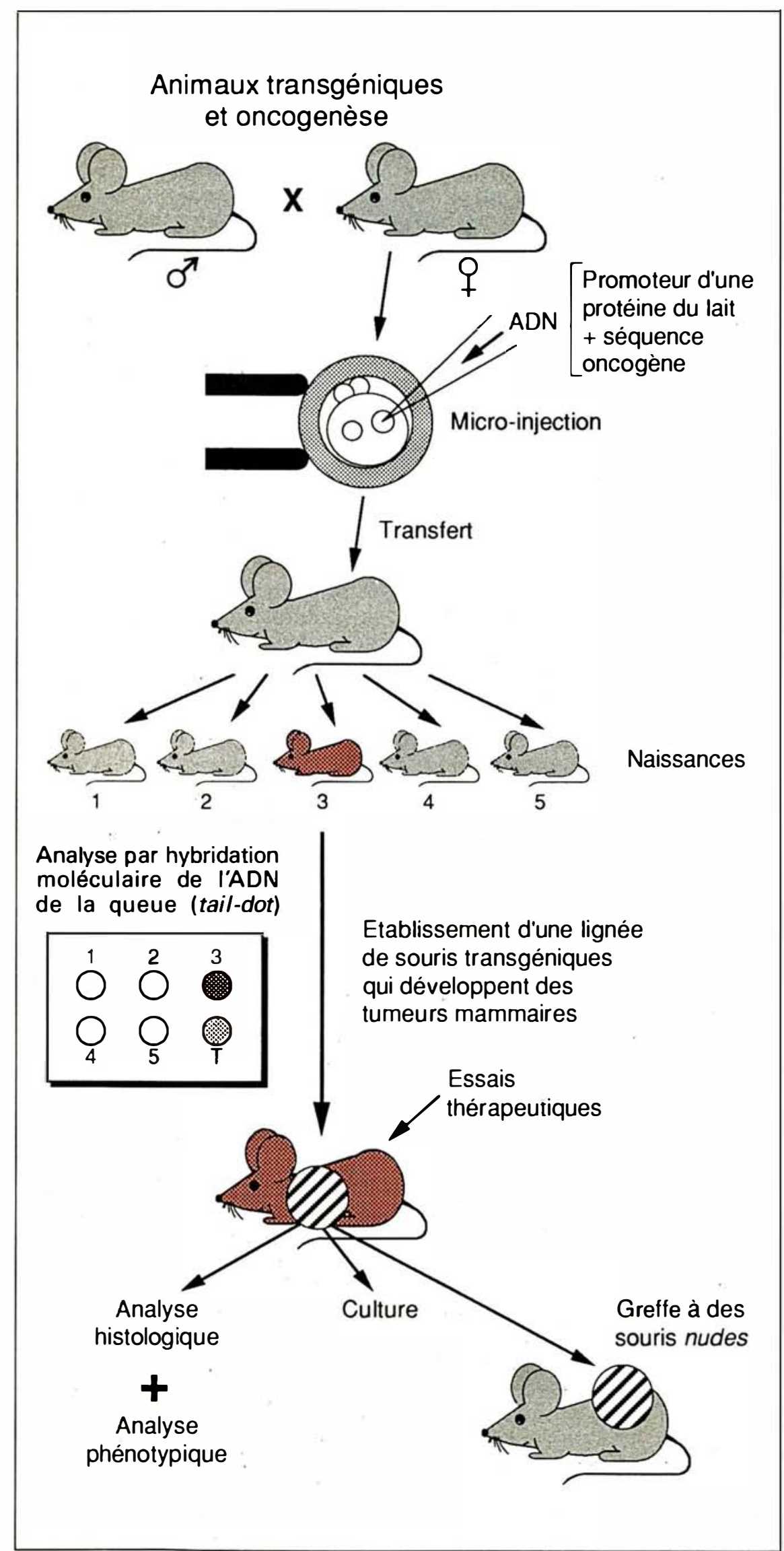

$m / s n^{\circ} 3$ vol. 5 , mars 89
Figure 1. Approche méthodologique : exemple de ciblage de l'expression d'un oncogène dans les cellules épithéliales de glando mammaire. Les différentes étapes de production et d'analyse des souris transgéniques sont représentées. AnaIyse histologique: effet de l'expression d'un ou de plusieurs oncogènes sur la croissance et la différenciation des cellules épithéliales en fonction de l'imprégnation hormonale de l'animal: gestation, lactation; phénomènes de régression après la lactation (persistance de foyers d'hyperplasie ?) ; formation et progression des tumeurs. Culture: tentatives pour établir des lignées cellulaires transformées. Analyse phénotypique : état de différenciation fonctionnelle des cellules transformées lexpression des transgènes, des gènes des protéines du lait, d'autres protooncogènes...): dépendance ou indépendance de cette différenciation vis-à-vis des conditions hormonales. Cette analyse est effectuée de manière comparative entre les cellules tumorales initiales, les lignées cellulaires dans le cas de leur obtention, les tumeurs apres transplantation dans des souris nudes. Greffe à des souris nude : permet d'anaIyser les rapports et les interactions entre les cellules transformées et les cellules, en particulier conjonctives, de I'hôte. Essais thérapeutiques: pharmacologie de l'action de divers antimitotiques, conditions d'apparition de lignées cellulaires résistantes, recherche de marqueurs cellulaires qui puissent être utilisés en cytophotométrie de flux", essai de ciblage de toxines, recherche de marqueurs de gravité et de pronostic évolutif.
* Détection d'un marqueur cellulaire (de membrane intracellulaire) au niveau des cellules individuelles appartenant $\grave{a}$ un flux continu de cellules. Le plus souvent réalisée à l'aide de faisceaux laser, cette méthode permet d'étudier la distribution d'un marqueur dans un ensemble complexe de cellules; elle peut également, couplée à un trieur de cellules, être utilisée pour séparer des cellules selon leur expression d'un (parfois de deux) marqueur(s). 


\section{RÉFÉRENCES}

24. Schönenberger CA, Andres CA, Groner B, Van der Valk M, Lemeur M, Gerlinger P. Targeted $c-m y c$ expression in mammary glandes of transgenic mice induces tumors with constitutive milk protein gene transcription. EMBO J 1988 ; 7 : 169-75.

25. Andres AC, Van der Valk M, Schönenberger CA, et al. Ha-ras and $c-m y c$ oncogene expression distinctly interferes with morphological and functional differentiation of mammary epithelial cells in single and double transgenic mice. Genes Dev 1988 ; 2 : 1486 95.

26. Brinster RL, Chen HY, Messing A, Van Dyke T, Levine A J, Palmiter RD. Transgenic mice harboring SV40 T-antigen genes develop characteristic brain tumors. Cell 1984 ; 37: 367-79.

27. Hanahan D. Heritable formation of pancreatic beta-cell tumors in transgenic mice expressing recombinant insulin/simian virus 40 oncogenes. Nature $1985 ; 315$ : 115-22.

28. Quaife CJ, Pinkert CA, Ornitz DM, Palmiter RD, Brinster RL. Pancreatic neoplasia induced by ras expression in acinar cells of transgenic mice. Cell $1987 ; 48: 1023-34$

29. Mahon KA, Chepelinsky AB, Khillian JS, Overbeek PA, Platigorsky J, Westphal $\mathrm{H}$. Oncogenesis of the lens in transgenic mice. Science 1987 ; 235 : 1622-8.

30. Messing A, Chen HY, Palmiter RD, Brinster RL. Peripheral neuropathies, hepatocellular carcinomas and islet cell adenomas in transgenic mice. Nature 1985 ; 316 : 461-3. 31. Field LJ. Atrial natriuretic factor-SV40 T antigen transgenes produce tumors and cardiac arrhythmias in mice. Science 1988 ; 239 : 1029-33.

32. Kelly F Kellermann O, Mechali F Gaillard J, Babinet C. Expression of SV40 oncogenes in F9 embryonal carcinoma cells, in transgenic mice and in transgenic embryos. Cancer cell, vol. 4 DNA Tumor Viruses. Cold Spring Harbor: Cold Spring Harbor Laboratory, $1988:$ 363-72.

33. Nusse $R$. The int genes in mammary tumorigenesis and in normal development. Trends Genet 1988 ; 4 : 291-5.

34. Rüther U, Garber C, Komitowski D Müller R, Wagner EF. Deregulated $c$-fos expression interferes with normal bone development in transgenic mice. Nature 1987 ; 325 : 412-6.

35. Willams RL, Courtneidge SA, Wagner EF. Embryonic lethalities and endothelial tumors in chimeric mice expressing polyma virus middle $\mathrm{T}$ oncogene. Cell $1988 ; 52$ : 121 31.

36. Chen S, Botteri F, Van der Putten $H$, Landel CP, Evans GA. A lymphoproliferative abnormality associated with inapropriate expression of the Thy-l antigen in transgenic mice. Cell $1987: 51$ : 7-19.

37. Lacey S, Alpert S, Hanahan D. Bovine papillomavirus genome elicits skin tumors in transgenic mice. Nature $1986 ; 322$ : 609-12.

38. Vogel J, Hinrichs SH, Reynolds RK, Luciw PA, Jay G. The HIV tat gene induces dermal lesions resembling Kaposi's sarcoma tous tissus exprimant largement le transgène [19]. Si les tumeurs obtenues sont intéressantes pour essayer de définir les événements supplémentaires à l'expression de myc qui sont nécessaires à l'apparition des tumeurs, ce système présente le désavantage de ne pas cibler l'expression de $m y c$ dans un type cellulaire particulier, encore moins à une étape précise de leur différenciation. L'expression de $m y c$ peut en effet débuter très tôt, à un moment non précisé de la cytodifférenciation ou de la maturation physiologique des organes intéressés, en raison de la synthèse de glucocorticoïdes par les surrénales fœetales. C'est pourtant ce système qui a été choisi initialement pour rechercher une synergie d'action des oncogènes Ha-ras et $c$-myc, dont on sait qu'elle est suffisante pour transformer des fibroblastes de rongeur en culture primaire [20], synergie d'action analysée également pour d'autres oncogènes [21]. L'oncogène $H$ ras est plus exprimé dans les tissus embryonnaires et fotaux que dans les tissus adultes. La protéine correspondante est impliquée dans la transmission des informations de la membrane cellulaire vers le noyau, son expression peut donc, selon les cas, soit inhiber soit promouvoir la différenciation cellulaire. L'oncogène ras activé par mutation est retrouvé et impliqué dans la formation de 10 à $20 \%$ des tumeurs humaines. Le transgène $\mathrm{MMTV} / \mathrm{Ha}$ ras s'exprime dans les mêmes tissus que $M M T V / c-m y c$, causant des adénocarcinomes mammaires précoces et envahissants, tant chez le mâle que chez la femelle, et des tumeurs lymphocytaires, mais également des tumeurs salivaires, ce qui n'était pas le cas pour $M M T V / c-m y c$. Le croisement de souris $M M T V / c-m y c \times$ MMTV/Ha-ras permet d'obtenir des animaux doublement transgéniques chez lesquels les tumeurs mammaires se développent extrêmement précocément, démontrant effectivement qu'un des événements favorisant la transformation cellulaire peut être l'activation d'un second oncogène [22]. En collaboration avec le Ludwig Institute for Cancer Research de Berne, nous avons obtenu des résultats similaires par ciblage de l'expression des mêmes oncogènes spécifiquement dans les cellules épithé- liales mammaires dont l'activité est sous contrôle des hormones lactogènes [23-25]. Ces oncogènes vont exercer une profonde influence sur la croissance, la différenciation et la maturation physiologique des cellules dans lesquelles ils s'expriment. L'oncogène ras retarde la différenciation qui s'effectue normalement au cours de la gestation, sans avoir d'effet sur la phase finale de formation des acini pendant la lactation, ni leur régression après celle-ci. L'oncogène $m y c$, au contraire, bloque les cellules épithéliales à un stade précoce de la phase proliférative qui marque le début de la différenciation, il restera de ce fait de nombreux îlots d'hyperplasie après la fin de la lactation. In vivo, les cellules épithéliales transformées qui expriment myc synthétisent des protéines du lait et induisent la synthèse d'une protéine particulière de la matrice extracellulaire (tenascine) normalement présente uniquement pendant la vie fœtale, au moment où les interactions entre cellules épithéliales et mésenchymateuses permettent l'apparition de l'ébauche mammaire. Ces mêmes cellules, in vitro, n'expriment plus ni le transgène ni les protéines du lait. En revanche, elles reprennent toutes ces fonctions et forment des tumeurs dès lors qu'elles sont greffées sous la peau de souris nude. Ces expériences montrent ainsi l'importance de la réaction des cellules voisines des cellules épithéliales pour la constitution d'une tumeur, réponse qui n'est pas identique selon l'oncogène exprimé.

La croissance tumorale in vivo semble donc nécessiter l'activation de plusieurs oncogènes. Mais ce n'est pas toujours le cas. Il existe une corrélation nette entre l'expression de l'oncogène $c$-erbB-2 (ou $c$-neu) et la progression clinique des cancers du sein. Cet oncogène a été initialement isolé à partir d'un neuroblastome induit chimiquement chez le rat. La protéine correspondante est apparentée au récepteur du facteur de croissance épidermique, et son activation est liée à la substitution d'un seul acide aminé dans le domaine transmembranaire. L'expression du transgène $M M T V / c$-neu [12] entraîne un défaut de lactation, une hyperplasie massive de tout l'épithélium et l'apparition syn- 
chrone de nombreuses tumeurs dans toutes les glandes mammaires des souris transgéniques. Il semble donc bien ici que l'expression d'un seul oncogène soit suffisante pour entrâ̂ner la transformation d'un très grand nombre de cellules mammaires. Inversement, l'expression du même transgène dans la parotide ou l'épididyme ne cause qu'une hyperplasie bénigne.

\section{Conclusions et perspectives}

Quelles conclusions et quel avenir pour ces modèles expérimentaux ? Les souris utilisées en laboratoire développent rarement les types tumoraux que font invariablement, et après un temps de latence caractéristique, les animaux transgéniques pour divers oncogènes. Ils ont donc acquis une prédisposition particulière et l'expression, plus ou moins ciblée, des oncogènes est une condition nécessaire mais non suffisante à l'obtention des tumeurs. Le premier d'une série d'événements aboutissant à la transformation cancéreuse est donc accessible expérimentalement. Ces modèles devraient permettre d'appréhender les maillons manquants. Quelques paramètres se dégagent.

- Le taux d'expression d'un oncogène. Le ciblage de myc dans les lymphocytes B entraîne la formation de lymphomes après ll semaines avec le enhancer des chaînes lourdes d'immunoglobuline et après 40 semaines seulement avec celui du gène de la chaîne légère $K$; cette différence reflète les niveaux d'expression des transgènes obtenus avec ces deux enhancers.

- La dépendance du type cellulaire. L'oncogène myc est un «bon » oncogène pour les lymphocytes ou les cellules épithéliales mammaires. Il est cependant sans effet sur les cellules épithéliales pancréatiques ou salivaires. Quelle est la distinction entre ces types cellulaires? Expriment-ils de manière naturelle des oncogènes ou d'autres facteurs susceptibles ou non de complémenter l'action de myc? Tout ou partie des gènes dont l'expression est normalement contrôlée par $m y c$ sont-ils dans une configuration chromatinienne non accessible dans les cellules pan$\mathrm{m} / \mathrm{s} n^{\circ} 3$ vol. 5 , mars 89 créatiques et salivaires? La voie est ouverte pour la compréhension de ces phénomènes.

L'intérêt des souris transgéniques ne s'arrête pas là, et plusieurs applications surgissent immédiatement à l'esprit.

- Lignées cellulaires. Cette capacité des oncogènes d'induire l'immortalisation, et donc la prolifération cellulaire, devrait faciliter l'établissement de lignées cellulaires en culture à partir de types cellulaires peu ou transitoirement représentés pendant l'ontogenèse. Seule une fraction restreinte des types cellulaires des mammifères existe sous forme de lignées cellulaires accessibles. Cependant pérenniser de telles lignées - qui doivent garder leurs caractéristiques (expression de protéines spécifiques) et pour lesquelles il faut définir des conditions de culture adéquates est un travail long et difficile.

- Modèles de vaccination. Le BPVI (bovine papilloma virus) infecte les tissus cutanés des bovins. Les séquences oncogènes de ce virus entraînent la formation de fibropapillomes composés de fibroblastes et de kératinocytes. Les séquences du BPVI introduites dans le génome de souris transgéniques induisent la formation de tumeurs de la peau dans les régions exposées [37]. Ceci signifie soit que les éléments régulateurs du BPV sont correctement reconnus, les oncogènes viraux ne s'exprimant que dans les cellules cutanées de souris, soit que le BPV s'exprime en fait dans toutes les cellules murines, seules celles de la peau étant transformables par ces oncogènes viraux. Un modèle analogue, utilisant le HPV par exemple (human papilloma virus), devrait permettre la mise au point de stratégies de vaccination antitumorale, dont l'importance est évidente si l'on songe au cancer du col de l'utérus, par exemple.

- Essais thérapeutiques. En toute logique l'analyse des événements survenant lors de la transformation cellulaire des animaux transgéniques permettra la mise en évidence de marqueurs spécifiques des cellules tumorales, ce qui pourrait conduire à l'établissement de modèles expérimentaux pour cibler l'action, par exemple de toxines, dans le but de détruire spécifiquement les cellules cancéreuses

\section{Summary}

\section{Oncogenesis in transgenics}

Transgenic mice have been used to demonstrate that normal cellular genes (proto-oncogenes) if deregulated can cause cancer. Lines of mice developping specific tumor types because of targeted expression of distinct oncogenes are now avaible. The effects of oncogene protein production on growth, differentiation and transformation can be analyzed as a function of time of expression, quantity and cell type in which they are expressed. Moreover it is possible to maintain genes determining different events in the induction and progression of specific tumors as transgenes in separate lines which can be mated. Therefore the specific roles played by different oncogenes and/or growth factors in tumor formation and evolution can be analyzed. Transgenic animals bearing deregulated oncogenes represent a good oportunity for developping lines from rare cell types and models for vaccination or therapeutic assays.

\section{TIRÉS A PART}

P. Gerlinger. 